\title{
Optical vortex generation by volume holographic elements with embedded phase singularity: Effects of misalignments
}

\author{
${ }^{1}$ Bekshaev A., ${ }^{2}$ Sviridova S., ${ }^{1}$ Popov A., ${ }^{1}$ Rimashevsky A. and ${ }^{1}$ Tyurin A. \\ ${ }^{1}$ Research Institute of Physics, I. I. Mechnikov National University, 2 Dvorianska \\ Street, 65082 Odessa, Ukraine, e-mail: bekshaev@onu.edu.ua \\ ${ }^{2}$ Institute of Computer Systems, Odessa National Polytechnic University, \\ Shevchenko Avenue 1, 65044 Odessa, Ukraine, \\ e-mail: sviridovasvetlana@gmail.com
}

Received: 28.06 .2013

\begin{abstract}
Based on the linear theory for optical vortex (OV) formation in volume holographic elements (HE) with embedded phase singularity (A. Bekshaev et al., Opt. Commun. 285 (2012) 4005), we analyze theoretically the OV-beams obtained when the incident Gaussian beam axis deviates from the optical axis of the HE. For different displacements of the incident beam with respect to the HE centre, the spatial characteristics of the diffracted beams and their evolution during the post-HE propagation are investigated numerically with allowance for the radiation extinction in the HE depth. A special attention is paid to behaviour of the beam centroid (centre of gravity) trajectory. The sensitivity of the generated OV-beam profile to the incident beam misalignments can be used for the output beam shaping and control, in particular, for compensation of the OV-beam distortions associated with the light extinction.
\end{abstract}

Keywords: optical vortex, volume hologram, misalignment, transformation, spatial structure, beam propagation

PACS: 42.25.Bs, 42.25.Fx, 42.40.Pa, 42.40.Eq, 42.50.Tx, 42.60.Jf, 42.90.+m

UDC: $535.41+535.42 / .44$

\section{Introduction}

Optical vortices (OVs) are regions with a circular motion of energy in the electromagnetic wave [1]. The energy circulation is closely associated with the optical field singularities. In the important case of paraxial light beams with OVs, a transverse circulatory flow is typical, in addition to the main (longitudinal) energy transportation [2]. Accordingly, the transverse profile of such beams contains one or more singular points with zero amplitude and indefinite phase; upon a round trip near such a point, the field phase grows by $2 \pi m$ where $m$ is an integer topological charge (order) of the OV that forms a so-called screw wavefront dislocation $[1,2]$.

Beams with the OVs reveal many useful properties which make them promising for a lot of practical applications: capturing and manipulation of microparticles [3-5], measurement of small displacements and exact localization of optical inhomogeneities [6-9], as well as improving resolution of spatially overlapping optical signals [10-13] and encoding or processing information [14-16]. This entails a necessity for regular and reliable means of their generation.

Among diverse approaches known for the generation of OV-beams [1, 2, 17-20], the most suitable and efficient ones are based on holographic principles [21-23], in which a beam passes through a special holographic element (HE) [24, 25]. Such HEs reproduce the patterns of interference between a 'regular' (vortexless) and singular beams. Their spatial configuration resembles a 
diffraction grating with one or several broken grooves, forming a so-called 'fork' structure [24, 25, 26] that represents an embedded phase singularity (EPS) associated with the hologram. If a vortexless beam falls onto the centre (i. e, the groove bifurcation point - see Fig. 1) of such a hologram, diffracted beams of all orders except the zero-order (non-diffracted) beam acquire a vortex structure.

The OV-beams obtained by means of HEs with EPSs are characterized by a particular set of characteristics. Their properties have been studied in many experimental and theoretical works $[17,24,25-36]$, though practically all of the results are limited to the case of a thin (plane) HE (in this case the generated OV-beams can be united within a special class of 'Kummer beams' [30], or 'hypergeometric-Gaussian beams' [34]). However, operation of such thin HEs is not free from essential drawbacks: simultaneous diffraction to a number of orders leads to low intensities of any of the individual diffracted beams, each diffracted OV-beam is rather sensitive to uncontrollable conditions of HE-induced transformations, and the whole OV-generation procedure suffers from low angular and spectral selectivities. At least, a portion of these shortcomings can be eliminated using volume (or thick) HEs which can be formed via recording a 3D interference pattern of a standard OV-carrying Laguerre-Gaussian beam and a reference Gaussian vortexless beam [37]. Additionally, it has been shown that, apart from the improved selectivity and diffraction efficiency, the volume HEs with EPSs provide some new possibilities for purposeful formation of OV-beam spatial profiles, which are attractive for many OV-generation techniques.

At the same time, the value of the results known for the operation of volume HEs is rather limited as they relate predominantly to the ideal ('nominal') beam transformation scheme, where the incident beam axis coincides exactly with the axis of the reference beam used for recording the HE. Such conditions prevent one from analyzing the consequences of possible misalignments in the mutual disposition of the incident beam and the OV-generating HE with the EPS. However, in practice the HE fabrication and the arrangement of any optical system in which it is used bring about inevitable deviations from ideal pre-specified configurations. Besides, the properties of the OV-beams obtained under conditions of HE misalignment can be profitably exploited. For the OV-beams generated with thin HEs, this has been demonstrated for metrological and astronomical applications [9-13], as well as for discriminating different OV-beams in systems of information transfer and processing [14-16, 38-41]. All of these points make it highly relevant to study in detail the role and significance of the diffracted-beam distortions appearing due to nonideal arrangement of the incident beam and the HE the with EPS. A general approach to this problem has been developed in application to thin HE $[33,36]$. In the present work, we generalize it and apply to the OV-beams generated by thick HEs, basing on the theory developed earlier for perfectly aligned volume HEs with EPSs [37].

The article starts with reproducing a general scheme of analysis of the HE with EPS [37] in the form suitable for studying the misalignment effects (Section 2). The results of numerical simulations of spatial properties of the diffracted beam are presented in Section 3. The general consideration of the OV-beam intensity profile with account for non-nominal configuration of the transformation system is followed by detailed investigation of the diffracted-beam centre of gravity as a suitable and universal characteristic of the light beam trajectory. Finally, the results are briefly discussed and their significance is estimated in Conclusion.

\section{Problem formulation}

For describing the OV-beams produced by thick HEs with EPSs, we employ a simple linear theory [37] restricted to a single-scattering model. It does not take into account the incident field varia- 
tions upon passing the hologram depth, and the whole HE is treated as a sequence of thin layers parallel to its input face. The resultant diffracted field appears as a superposition of secondary waves formed independently due to the incident wave diffraction by consecutive thin layers of the recording medium.

Following Ref. [37], we consider the action of a thick HE with EPS (see Fig. 1). Here, the HE-associated frame of reference $(x, y, z)$ is introduced, with the origin at the HE centre so that the coordinate plane $z=0$ is situated in the middle of the hologram depth and is parallel to its input face. This plane divides the HE into two equal parts, the forward $(-d<z<0)$ and backward $(0<z<d)$ ones (see Fig. 1). The forward part of the HE is conventionally moved down and the backward one is shown in detail (note also differing positions and appearances of the 'forks' in the central and input planes of the HE). To describe the diffracted wave, we employ the additional Cartesian frame of reference $\left(x_{2}, y_{2}, z_{2}\right)$. The origins of the both coordinate systems coincide with the bifurcation point located in the central transverse plane $z=0$. The axes $z_{1}$ and $z_{2}$ indicate the nominal directions of the incident and diffracted beams, respectively; the axes $y$ and $y_{2}\left(x\right.$ and $x_{2}$ ) are parallel (orthogonal) to the HE grooves far from the hologram centre; the axis $x_{2}$ belongs to the cross section of the diffracted beam. Instead of the fringes forming a thin HE, the volume HE grooves are 3D structures elongated inside the $\mathrm{HE}$ at a certain angle $\theta_{t}$ with respect to the $\mathrm{HE}$ axis $z$. This angle is determined by directions of the recording waves, both of them lying in the horizontal plane: if the reference Gaussian beam and the object OV-beam cross the HE input plane respectively at the angles $\theta_{r}$ and $\theta_{s}$, then we have $\theta_{t}=\left(\theta_{r}+\theta_{s}\right) / 2$ [37]. All of the incidence angle $\theta_{1}$, the groove orientation angle $\theta_{t}$ and the diffraction angle $\theta_{2}$ lie in the horizontal planes parallel to the plane $x z$.

The nominal recording $\left(\theta_{r}\right.$ and $\left.\theta_{s}\right)$ and readout $\left(\theta_{1}\right.$ and $\left.\theta_{2}\right)$ angles are linked with the relations [37]

$$
\begin{aligned}
& \left(\sin \theta_{1}-\sin \theta_{2}\right)-m\left(\sin \theta_{r}-\sin \theta_{s}\right)=0 \\
& \left(\cos \theta_{1}-\cos \theta_{2}\right)-m\left(\cos \theta_{r}-\cos \theta_{s}\right)=0,
\end{aligned}
$$

where $m$ is the order of diffraction. Following the work [37], we accept that

$$
m=1, \quad \theta_{1}=\theta_{r}=0, \quad \theta_{2}=\theta_{s}=2 \theta_{t},
$$

which implies that the nominal incident beam axis $z_{1}$ coincides with the HE axis $z$. In the present work we consider only a monochromatic (wavenumber $k=2 \pi / \lambda$ ) paraxial readout beam, whose optical field can be described as $u(x, y, z) \exp (i k z)$, with slowly varying complex amplitude $u(x, y, z)$. Then the diffracted beam evolves as a paraxial beam with the nominal axis $z_{2}$ and, quite similarly, it can be described in the associated frame of reference $\left(x_{2}, y_{2}, z_{2}\right)$ as $u_{2}\left(x_{2}, y_{2}, z_{2}\right) \exp \left(i k z_{2}\right)$ [32,37]. For a further detailed analysis, we take the situation where the incident beam is Gaussian and approaches the central plane of a hologram in its waist plane, where the wavefront is flat.

The system misalignments are implemented by means of displacements of the incident beam such that its axis crosses the central plane of the HE at the point $\left(s_{x}, s_{y}\right)$ and its direction deviates from the nominal $z$ axis by small angles $\left(g_{x}, g_{y}\right)$ (see Fig. 1). Further on, it would be suitable to express all the transverse coordinates and the quantities characterizing the beam cross sections in 
the units of the incident beam radius $b$ measured at the level $e^{-1}$ of the maximum intensity,

$$
(x, y) \rightarrow \frac{(x, y)}{b}, \quad\left(x_{2}, y_{2}\right) \rightarrow \frac{\left(x_{2}, y_{2}\right)}{b}, \quad\left(s_{x}, s_{y}\right) \rightarrow \frac{\left(s_{x}, s_{y}\right)}{b},
$$

the longitudinal distances - in units of the corresponding Rayleigh range $z_{R}=k b^{2}$,

$$
\left(z, z_{2}\right) \rightarrow \frac{\left(z, z_{2}\right)}{k b^{2}},
$$

and all the angular deviations - in the units of the incident beam self-diffraction angle $(\mathrm{kb})^{-1}$,

$$
\left(g_{x}, g_{y}\right) \rightarrow\left(g_{x}, g_{y}\right) k b .
$$

Since the influence of the volume HE on the spatial profile of the diffracted beam has been shown to be determined by the ratio between the hologram thickness and the incident beam radius [37], the characteristics of bulk nature of the HE (i.e., its half-thickness $d$ and the incident-beam amplitude extinction coefficient $\kappa$ ) will also be presented in the corresponding dimensionless forms:

$$
d \rightarrow \frac{d}{b}, \quad \kappa \rightarrow \kappa b .
$$

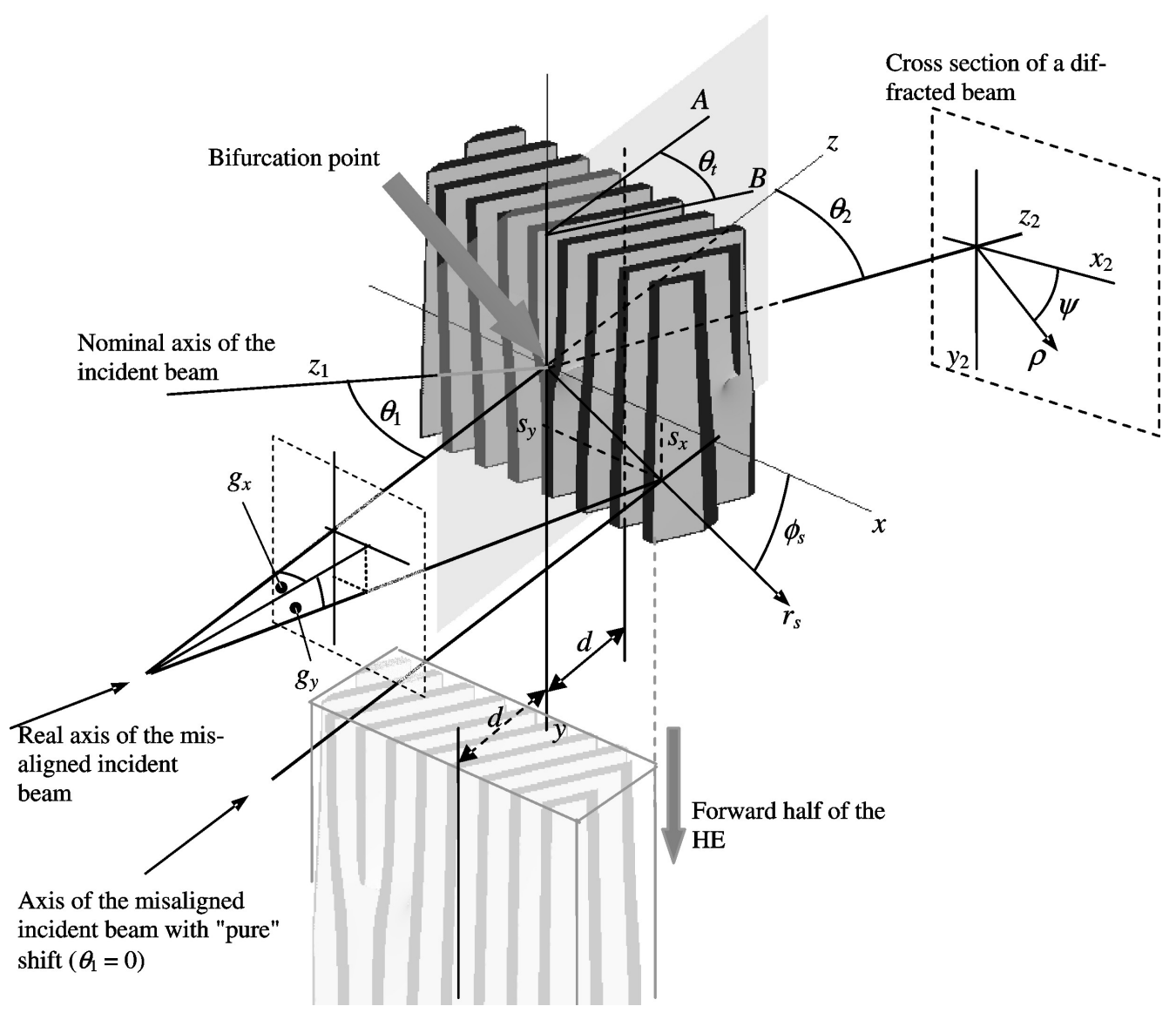

Fig. 1. Geometrical conditions of beam transformation in a volume HE with an EPS. A forward half of the HE is conventionally moved down and denudes the middle plane $z=0$; a semitransparent plane $x=0$ contains the $\mathrm{HE}$ axis $z$ orthogonal to its face and the axis $A$ parallel to $z$; the axis $B$ is parallel to the interference grooves, the angles $\theta_{1}, \theta_{t}, \theta_{2}$ and $g_{x}$ lie in horizontal planes, the angle $g_{y}$ lies in a vertical plane (see also the text). 
Under these conventions, a misaligned incident Gaussian beam is described by the complex amplitude distribution [37]

$$
u_{1}(x, y, 0)=\exp \left[-\frac{\left(x_{1}-s_{x}\right)^{2}+\left(y_{1}-s_{y}\right)^{2}}{2}\right] \exp \left\{i\left[g_{x}\left(x-s_{x}\right)+g_{y}\left(y-s_{y}\right)\right]\right\},
$$

while the complex amplitude of the diffracted beam can be calculated via the equation [37]

$$
\begin{aligned}
& u_{2}\left(x_{2}, y_{2}, z_{2}\right)=\text { const } \frac{1}{2 \pi i z_{2}} \frac{1+\cos \theta_{2}}{2 \gamma \cos \theta_{2}} \times \\
& \exp \left\{-\frac{1}{2} g_{x}^{\prime 2}+i \frac{z_{2}}{2}\left(\alpha^{2} g_{x}^{2}+g_{y}^{2}\right)+i\left[g_{x} \alpha x_{2}^{\prime}+g_{y}\left(y_{2}^{\prime}-s_{y}\right)\right]\right\} \times \\
& \int \exp \left(-i \alpha g_{x}^{\prime} x\right) D\left(d, x, s_{x}\right) \exp \left[-\left(\frac{y-s_{y}}{\sqrt{2}}\right)^{2}\right] e^{i l \phi} \exp \left\{\frac{i}{2 z_{2}}\left[\left(x_{2}^{\prime}-x\right)^{2}+\left(y_{2}^{\prime}-y\right)^{2}\right]\right\} d x d y,
\end{aligned}
$$

where $\phi=\arctan (y / x)$ is the polar angle in the coordinate plane $(x, y), l$ the topological charge of the EPS (in Fig. 1, we have $l=1$ ),

$$
\begin{gathered}
x_{2}^{\prime}=x_{2}-\alpha g_{x} z_{2}, \quad y_{2}^{\prime}=y_{2}-g_{y} z_{2}, \\
D\left(d, x, s_{x}\right)=\left[\operatorname{erf}\left(\frac{\alpha x+\gamma d-s_{x}-i g_{x}^{\prime}}{\sqrt{2}}\right)-\operatorname{erf}\left(\frac{\alpha x-\gamma d-s_{x}-i g_{x}^{\prime}}{\sqrt{2}}\right)\right],
\end{gathered}
$$

and $\operatorname{erf}(\tau)=\frac{2}{\sqrt{\pi}} \int_{0}^{\tau} e^{-t^{2}} d t$ is the error function [42]. The quantities

$$
\alpha=\left(\cos \theta_{2}\right)^{-1}, \quad \gamma=\tan \theta_{2}
$$

characterize the geometrical beam distortion due to propagation in the HE depth and the highangle diffraction [37], the 'complex angular deviation'

$$
g_{x}^{\prime}=g_{x}+i(\kappa / k \gamma)
$$

accounts for the incident beam attenuation while propagating within the thick HE, which is supposed to obey the Bouguer law $u_{1} \propto \exp [-\kappa(z+d)]$, where the HE depth $z+d$ and the amplitude extinction coefficient $\kappa$ are expressed in the units given by Eqs. (6). Eqs. (8)-(12) constitute a convenient basis for our further calculations and numerical simulations.

\section{Results and discussion}

Before starting the numerical evaluations, let us discuss some general points on how the misalignments affect the OV-beam generation. In the case of a thin HE [32, 33], the nominal conditions include the two requirements: (i) the incident beam axis is orthogonal to the HE plane, and (ii) the incident beam axis crosses the HE exactly at the bifurcation point. The requirement (i) is not critical as typically small angular deviations of the incident beam lead only to obvious geometrical transformations of the output beam direction. This allows one to reduce the whole problem to investigation of consequences of small parallel translations of the incident beam. For the volume HEs with EPSs, simple geometrical transformations equivalent to those allowing for violation of the requirement (i) are expressed by pre-integral factors in the second line of Eq. (8). It is seen that pure redefining the spatial coordinates does not eliminate the problem of angular misalignments: at least, a horizontal deviation $g_{x}$ also enters the integral term of Eq. (8) and, thus, it influences the 
OV-beam spatial profile. However, because of the angular selectivity [37], the diffracted beam power rapidly degrades with growing $g_{x}$ and perceptible beam deformations due to nonzero $g_{x}$ appear to be coupled with rather low diffraction efficiency. This, again, allows us to ignore possible violations of the requirement (i) and so concentrate on the situations when $g_{x}=g_{y}=0$. Then the incident Gaussian beam is directed at the nominal angle but its axis intersects the HE central plane at the point with the coordinates $x=s_{x}$ and $y=s_{y}$ (see Fig. 1).

Further, in case of a thin HE with EPS and small diffraction angles, the obvious asymmetry of the HE has manifested itself in the fact that the nominal directions of diffracted beams with different orders all lie in the horizontal plane $(x z)$, but has not affected the diffracted beam symmetry with respect to its own axis, which remains approximately circular [27, 28, 32]. In this context, the direction of transverse displacement of the incident beam has not been important: with respect to this direction, the diffracted beam deformation caused by the displacement has always been the same [33]. For the volume HEs, as well as for the thin HEs with high diffraction angles [36], the asymmetry of the diffracted beam profile cannot be neglected and this implies different responses of the diffracted beam profile to the $x$ - and $y$-oriented misalignments.

For detailed numerical analysis, we choose the same HE structure as used before [37]. It is characterized by the diffraction conditions given by Eq. (2), the EPS topological charge $l=1$, while the recording and readout angles are

$$
\theta_{1}=\theta_{r}=0, \quad \theta_{2}=\theta_{s}=\pi / 4
$$

In this situation the nominal incident beam axis coincides with the axis $z$ and the only type of the scheme misalignment is the incident beam parallel translation $(0,0) \rightarrow\left(s_{x}, s_{y}\right)$ (see Fig. 1). Although the numerical results are expressed in the relative units given by Eqs. (3)-(6), we imply their applications to the readout beam with $b=0.02 \mathrm{~cm}$ and $k \approx 10^{5} \mathrm{~cm}^{-1}$ (here a He-Ne laser radiation is assumed, with $\lambda=632.8 \mathrm{~nm}$ ).

\subsection{Influence of misalignments on spatial profile of the diffracted beam}

We start with considering the $x$-oriented shifts, when the incident beam misalignment occurs within the plane of diffraction ( $s_{y}=0$ and $s_{x} \neq 0$ - see Fig. 1). Fig. 2 illustrates theoretical patterns of the transverse intensity profiles for the OV-beams obtained by the volume HE with the EPS for the extinction coefficient $\kappa=0.4$ (in the units of $b^{-1}$ ). All the figures represent the patterns seen against the beam propagation at the distance $z_{2}=z_{R}$ behind the HE.

Comparing the $1^{\text {st }}$ and $2^{\text {nd }}$ rows of Fig. 2, one can conclude that increasing HE thickness from $2 d \approx 0.1 b$ up to $2 d \approx b$ makes no practical influence on the spatial profile of the diffracted beam. The beam patterns are very similar to those observed in case of the first-order OV-beam generation by a thin HE with an EPS under conditions of high-angle diffraction [36]. That agrees with the readout conventions given by Eqs. (2) and (13). Notice that the Bragg's character of the diffraction under conditions of Fig. 2 (as confirmed by the considerable angular selectivity of the HE with $2 d$ $\approx b-$ see Fig. 3 of Ref. [1]) does not affect in practice the spatial profile of the diffracted beam. In case of no misalignment (the column 0 ), both for a thin and a thick HEs, the beam is squeezed in the diffraction plane simultaneously with formation of the OV: the expected bright ring is replaced by an oval with inhomogeneous brightness; this happens due to the symmetry breakdown of the beam which leads to apparent intensity redistribution inside the beam spot [43]. It is seen that the incident beam displacements produce changes in the spatial profile of the resulting beam: with growing shift, the asymmetry increases, and the opposite displacements entail quite opposite beam 
profile deformations. A characteristic feature of the beam patterns presented in the $1^{\text {st }}$ and $2^{\text {nd }}$ rows is a remarkable symmetry between the intensity distributions corresponding to equal though opposite shifts of the incident beam axis.

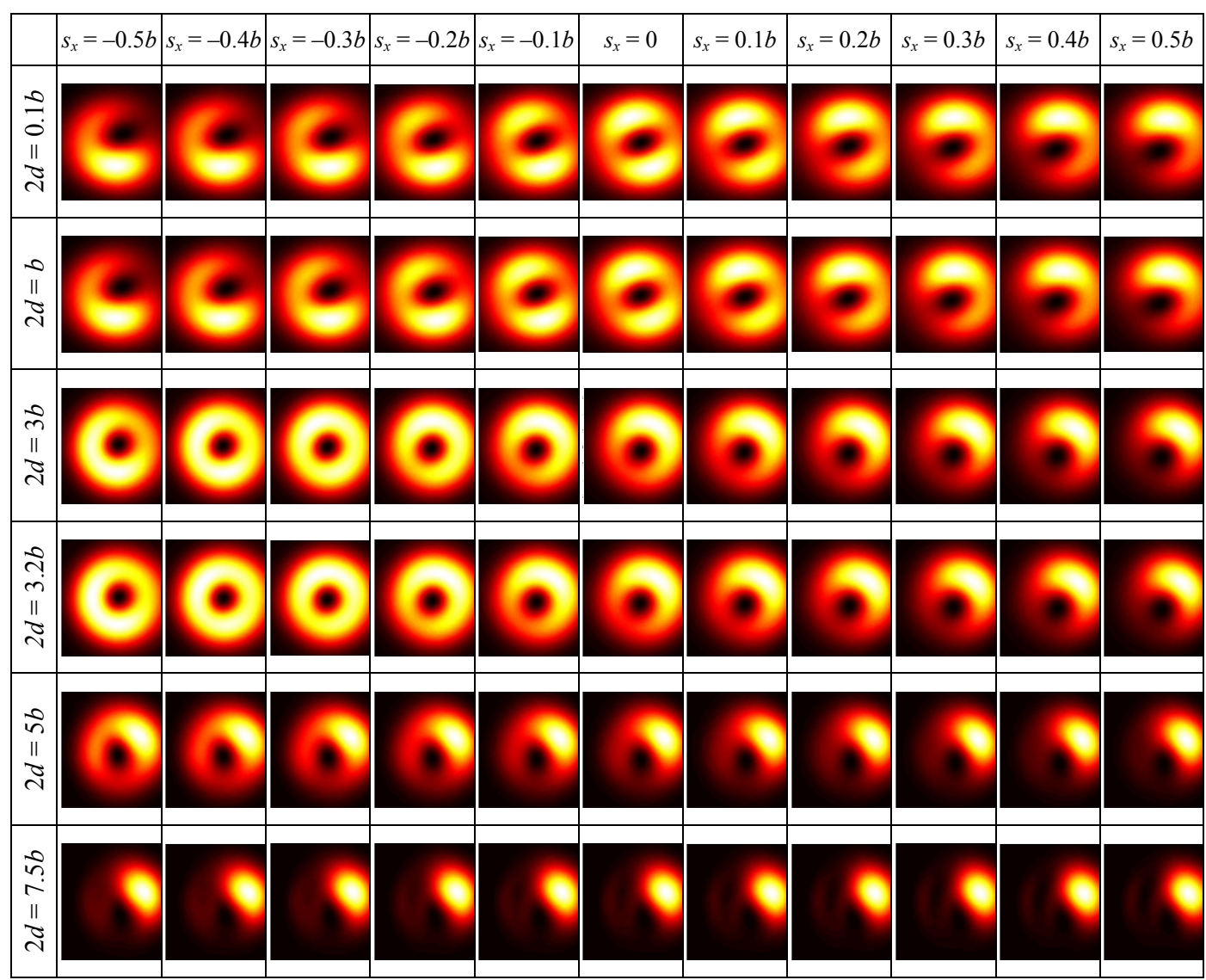

Fig. 2. Transverse intensity profiles of the OV-beams obtained after diffraction of Gaussian beam under the conditions given by Eqs. (11) and (13) in a volume HE with EPS, as calculated using Eq. (8) for the extinction coefficient $\kappa=0.4 b^{-1}$ and different displacements of the incident beam. The profiles are calculated for $z_{2}=z_{R}$ and zero vertical displacement $s_{y}=0$ (see Fig. 1). The horizontal displacements $s_{x}$ are indicated above each column, the HE thickness is indicated near each row, and the absolute size of the images (the length of the square side) is $7 b$.

With further increase in the HE thickness (see the $3^{\text {rd }}$ and the next rows of Fig. 2), the output beam shape appears to be asymmetrical even in the absence of any displacement $\left(s_{x}=0\right)$. This fact has been discussed in detail in Ref. [37] and is linked with a many-layer model of the HE: Different thin layers produce diffracted beams with mutual transverse shifts and of different powers, due to attenuation of the incident beam traversing the volume HE, and their superposition looks asymmetrical. Therefore, the attenuation manifests itself as a separate source of asymmetry of the diffracted beam, which acts jointly but independently of the hologram depth [37] and the misalignment. This allows one to find the conditions under which different sources compensate each other. Indeed, for the HE thicknesses $2 d=3 b$ and $3.2 b$, the diffracted beam profiles become more symmetrical and, when the incident beam is displaced by $s_{x}=-(0.3 \div 0.4) b$, the symmetrybreaking effect of the misalignment apparently disappears.

This fact inspires some interesting consequences: even in the conditions of high extinction, consistent with high diffraction efficiency of the volume HEs with the EPSs, deliberate misalign- 
ment can be used to produce the OV-beams with good circular symmetry. However, such a possibility occurs only when special relations between the extinction coefficient and the HE thickness hold true. With further increase in the HE thickness, the output beam loses its circular symmetry and no misalignment can restore it. Apparently, for such very thick HEs, the diffracted beam profile is almost exclusively determined by the incident wave attenuation, and the misalignment causes only weak visible changes to the transverse profile of the beam, and only at negative displacements $s_{x}$ (see the $5^{\text {th }}$ and $6^{\text {th }}$ rows of Fig. 2).

The case of orthogonal incident beam displacement $\left(s_{x}=0, s_{y} \neq 0\right.$, see Fig. 1) presented by Fig. 3 additionally exposes an $x-y$ asymmetry of the volume HE. The first two rows of Fig. 2 and Fig. 3 describe actually a thin HE with EPS. In fact, the images of Fig. 3 can be obtained by the rotation by $90^{\circ}$ of the corresponding images of Fig. 2, while the small residual differences can be ascribed to 'squeezing' of the images along the $x$ direction. For more thick HEs, the difference between Figs. 2 and 3 acquires a qualitative character. In particular, contrary to the $3^{\text {rd }}$ and $4^{\text {th }}$ rows of Fig. 2, no vertical displacement can compensate for a diffracted beam distortion induced by the HE thickness and extinction of the incident light. The images seen in the last rows of Fig. 2 and Fig. $3(2 d=5 b$ and $7.5 b)$ again become more similar, perhaps, because the influence of the hologram thickness and the light extinction is more essential for very thick HEs than the influence of any misalignment under consideration [37].

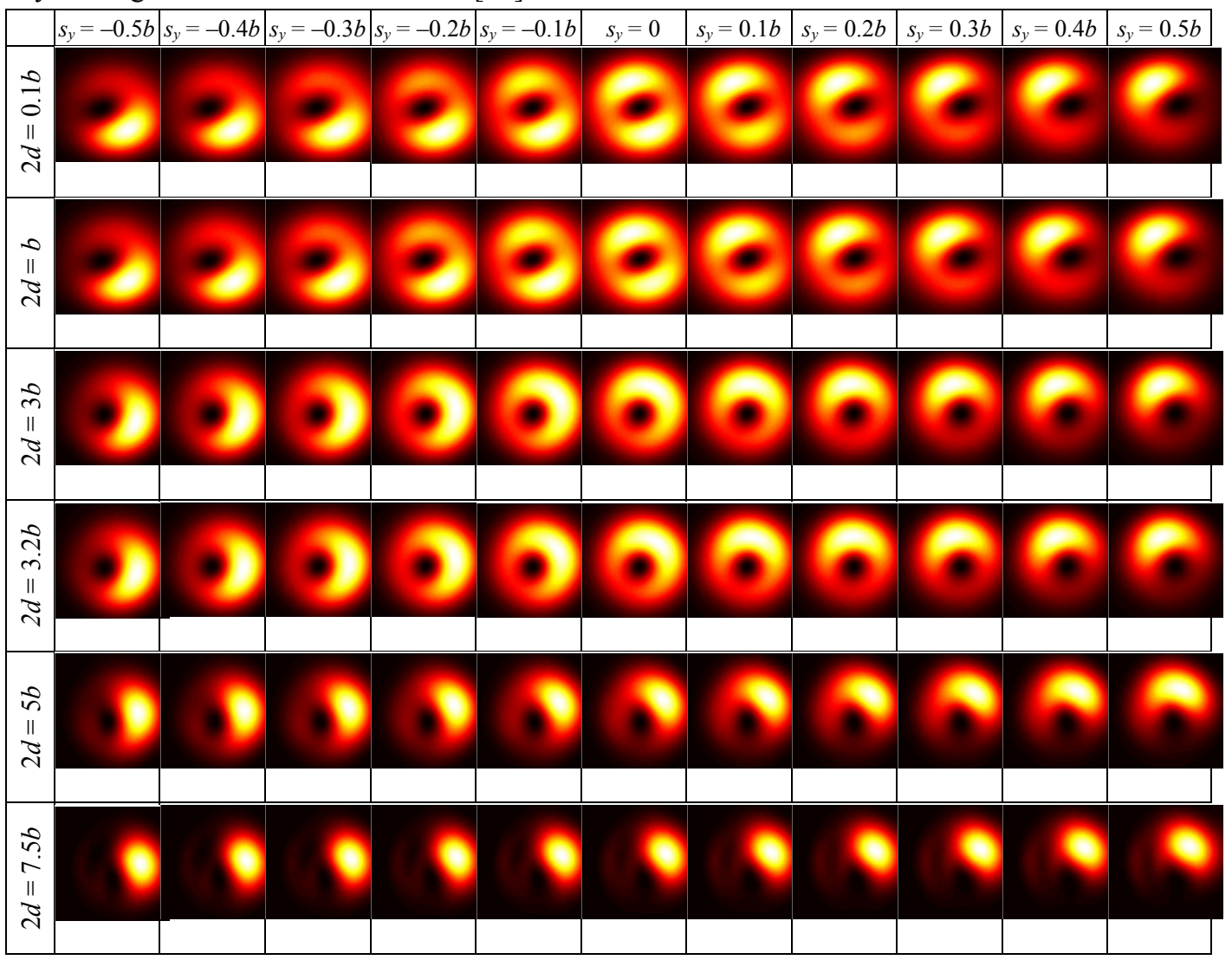

Fig. 3. The same as Fig. 2, but for zero horizontal displacement $S_{x}=0$. The vertical displacements $S_{y}$ are indicated above each column, the HE thickness is indicated near each row, and the absolute size of the images (the length of the square side) is $7 b$. 


\subsection{Displacements of the beam centre of gravity}

In a more detail, the spatial profile of the diffracted beam can be described in terms of the positions of certain special points. In the most common case, the intensity profile of the beam is characterized by the coordinates of centre of gravity (CG) of the beam $[33,36,44]$, which are defined by the relations

$$
\left\{\begin{array}{l}
x_{0}\left(z_{2}\right) \\
y_{0}\left(z_{2}\right)
\end{array}\right\}=\frac{\int\left\{\begin{array}{l}
x_{2} \\
y_{2}
\end{array}\right\}\left|u_{2}\left(x_{2}, y_{2}, z_{2}\right)\right|^{2} d x_{2} d y_{2}}{\int\left|u_{2}\left(x_{2}, y_{2}, z_{2}\right)\right|^{2} d x_{2} d y_{2}} .
$$

Here integration is performed over the whole cross section of the beam. The graphs seen in Figs. 4-6 represent the horizontal $\left(x_{0}\right)$ and vertical $\left(y_{0}\right)$ CG components as functions of the incident beam misalignment $\left(s_{x}, s_{y}\right)$. To interpret these results, we recall the well investigated case of a thin HE with EPS and small-angle diffraction [33, 44], which is called further on as a 'reference case'.

In that situation, the angular dependence of the output beam profile has been actually determined by the difference $\psi-\phi_{s}$, i.e. any beam deformation should be described with respect to the direction of misalignment (see Fig. 1). This means that, quite 'naturally', the beam profile's response on the misalignment should be characterized by the components of the CG displacement taken along and orthogonal to the misalignment axis:

$$
\begin{aligned}
& \xi_{0}=x_{0} \cos \phi_{s}+y_{0} \sin \phi_{s}=\rho_{0} \cos \left(\psi_{0}-\phi_{s}\right) \\
& \eta_{0}=-x_{0} \sin \phi_{s}+y_{0} \cos \phi_{s}=\rho_{0} \sin \left(\psi_{0}-\phi_{s}\right)
\end{aligned}
$$

where $\rho_{0}=\sqrt{x_{0}^{2}+y_{0}^{2}}$ and $\psi_{0}=a \arctan \left(y_{0} / x_{0}\right)$ are the polar coordinates of the CG, with $\rho_{0}$ and $\psi_{0}-\phi_{s}$ depending only upon the displacement magnitude $r_{s}=\sqrt{s_{x}^{2}+s_{y}^{2}}$. In case of a 'pure' $x$ oriented misalignment $\left(\phi_{s}=0\right)$, we have

$$
\xi_{0}\left(r_{s}, 0\right)=x_{0}\left(r_{s}, 0\right), \quad \eta_{0}\left(r_{s}, 0\right)=y_{0}\left(r_{s}, 0\right)
$$

(see the upper rows of Figs. 4-6). When the misalignment occurs along the $y$-direction $\left(\phi_{s}=\pi / 2\right)$, we have

$$
\xi_{0}\left(r_{s}, \pi / 2\right)=y_{0}\left(r_{s}, \pi / 2\right), \quad \eta_{0}\left(r_{s}, \pi / 2\right)=-x_{0}\left(r_{s}, \pi / 2\right)
$$

(see the lower rows of Figs. 4-6).

In the circularly symmetric reference case [33, 44], the conditions $\xi_{0}\left(r_{s}, \phi_{s}\right) \equiv \xi_{0}\left(r_{s}\right)=r_{s}$ and $\eta_{0}\left(r_{s}, \phi_{s}\right) \equiv \eta_{0}\left(r_{s}\right)$ have held with high accuracy, and there has been no difference between $\xi_{0}\left(r_{s}, 0\right)$ and $\xi_{0}\left(r_{s}, \pi / 2\right)$, or between $\eta_{0}\left(r_{s}, 0\right)$ and $\eta_{0}\left(r_{s}, \pi / 2\right)$. A comparison of the upper and lower rows of Figs. 4-6 shows that this difference is present for the volume HE. For relatively thin HEs (curves marked 0.1 and 1) with small extinction, the difference between the cases $\phi_{s}=0$ and $\phi_{s}=\pi / 2$ is comparatively small. It is only quantitative and can be ascribed to geometrical 'squeezing' of the profile of the diffracted beam due to high-angle diffraction (cf. with the corresponding beam-spot images shown in Fig. 4 of Ref. [37]). In particular, a noticeably lower steepness of the curves seen in panels (a), when compared to those seen in panels (c), is due to a reduced transverse size of the diffracted beam in the plane of diffraction. For the cases of high extinction (see Fig. 6, curves 3.2, 5 and 7.5), the responses of the CG to the $x$ - and $y$-oriented misalignments differ quali- 
tatively. This is a consequence of strong output-beam deformations associated with gradual attenuation of the incident beam upon its propagation within the HE (cf. with Fig. 5 of Ref. [37]).
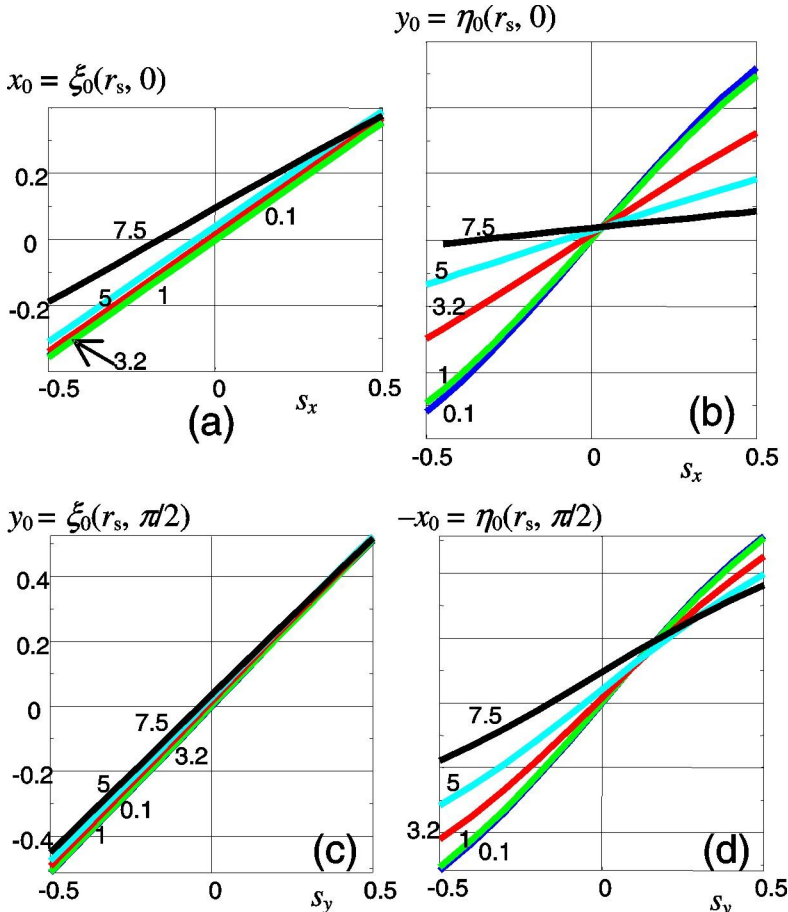

(c)

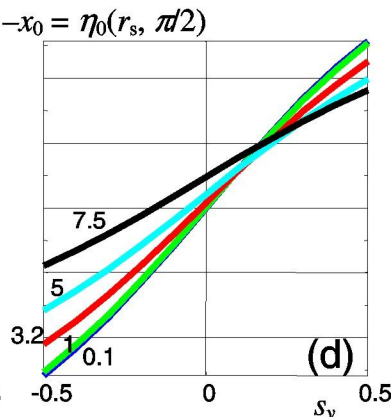

$s_{y} 0.5$

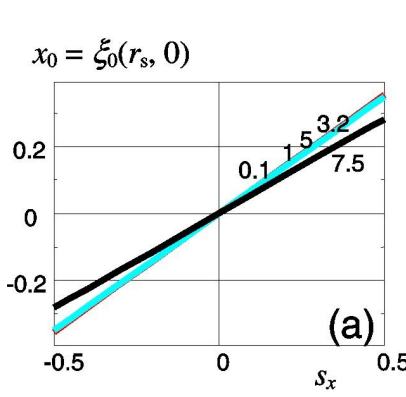

$y_{0}=\eta_{0}\left(r_{\mathrm{s}}, 0\right)$

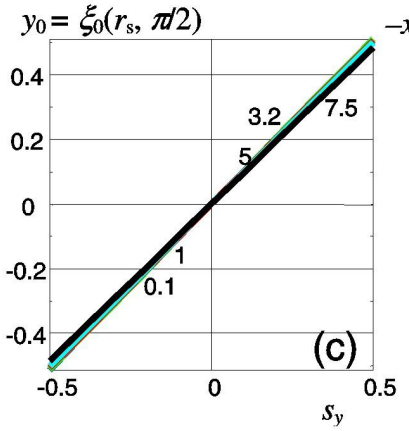

(c)
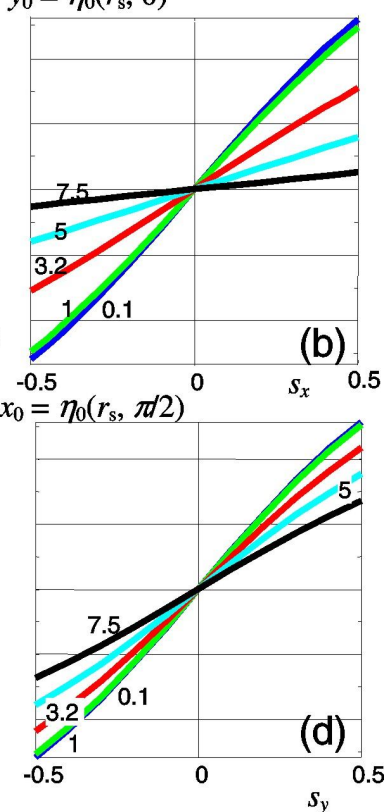

(b)
Fig. 4. CG coordinates of the diffracted beam profile for non-absorbing HE $(\kappa=0)$ vs. incident beam misalignment. Left column (a, c): CG shift component $\xi_{0}$ parallel to the incident beam displacement $\left(\psi=\phi_{s}\right)$; right column (b, d): CG shift component $\eta_{0}$ orthogonal to the incident beam displacement $\left(\psi=\phi_{s}+\pi / 2\right)$; upper row $(\mathrm{a}, \mathrm{b})$ : 'pure' $x$-misalignment $\left(s_{y}=0, s_{x}=r_{s} \cos \phi_{s}\right)$; lower row (c, d): 'pure' y-misalignment $\left(s_{x}=0\right.$, $\left.s_{y}=r_{s} \sin \phi_{s}\right)$. Each curve is marked by a corresponding $\mathrm{HE}$ thickness expressed in the units of $b$.

Fig. 5. The same as Fig. 4, but for the extinction coefficient $\kappa=0.02$. The curves for all the thicknesses practically coincide in panel (c)

For all of the cases consedered, the curves 0.1 and 1 are very close to each other, thus confirming that the volume character of HEs with EPSs does not affect the spatial structure of the diffracted beam till its thickness is less than the radius $b$ of the incident beam [37]. With further increase in the HE thickness and the extinction coefficient, the curves of the CG displacement along the misalignment, $\xi_{0}$, experience a parallel translation along the vertical direction, with almost no 
changes in their shapes (see curves 3.2, 5 and 7.5 in Fig. 6a). This is explained by extinctioninduced asymmetry of the diffracted beam (cf. Fig. 5 of Ref. [37]).

The CG behaviour for 'intermediately' directed misalignments is depicted in Fig. 7. Note that in the reference case of thin HEs $[33,44]$ there has been no need in special analysis of this situation because, since $\xi_{0}$ and $\eta_{0}$ do not depend on $\phi_{s}$, the CG coordinates for arbitrary $\phi_{s}$ could be determined by means of inverted Eqs. (15):

$$
x_{0}\left(r_{s}, \phi_{s}\right)=\xi_{0}\left(r_{s}\right) \cos \phi_{s}-\eta_{0}\left(r_{s}\right) \sin \phi_{s}, \quad y_{0}\left(r_{s}, \phi_{s}\right)=\xi_{0}\left(r_{s}\right) \sin \phi_{s}+\eta_{0}\left(r_{s}\right) \cos \phi_{s},
$$

or, with allowance for Eqs. (16) and (17), by the relations

$$
\begin{aligned}
& x_{0}\left(r_{s}, \phi_{s}\right)=x_{0}\left(r_{s}, 0\right) \cos \phi_{s}+x_{0}\left(r_{s}, \pi / 2\right) \sin \phi_{s}, \\
& y_{0}\left(r_{s}, \phi_{s}\right)=y_{0}\left(r_{s}, 0\right) \cos \phi_{s}+y_{0}\left(r_{s}, \pi / 2\right) \sin \phi_{s} .
\end{aligned}
$$

Fig. 7 demonstrates that the rule given by Eqs. (18) applies well for relatively thin HEs (see Fig. 7a-c) or even for thick HEs with low extinctions (see Fig. 7d, e). Only when a strong extinction is combined with a high HE thickness (see Fig. 7f), then, quite expectedly, the diffracted beam reaction on an arbitrarily directed misalignment cannot always be calculated as a simple superposition of the results obtained for the 'standard' situations with $\phi_{s}=0$ and $\phi_{s}=\pi / 2$.
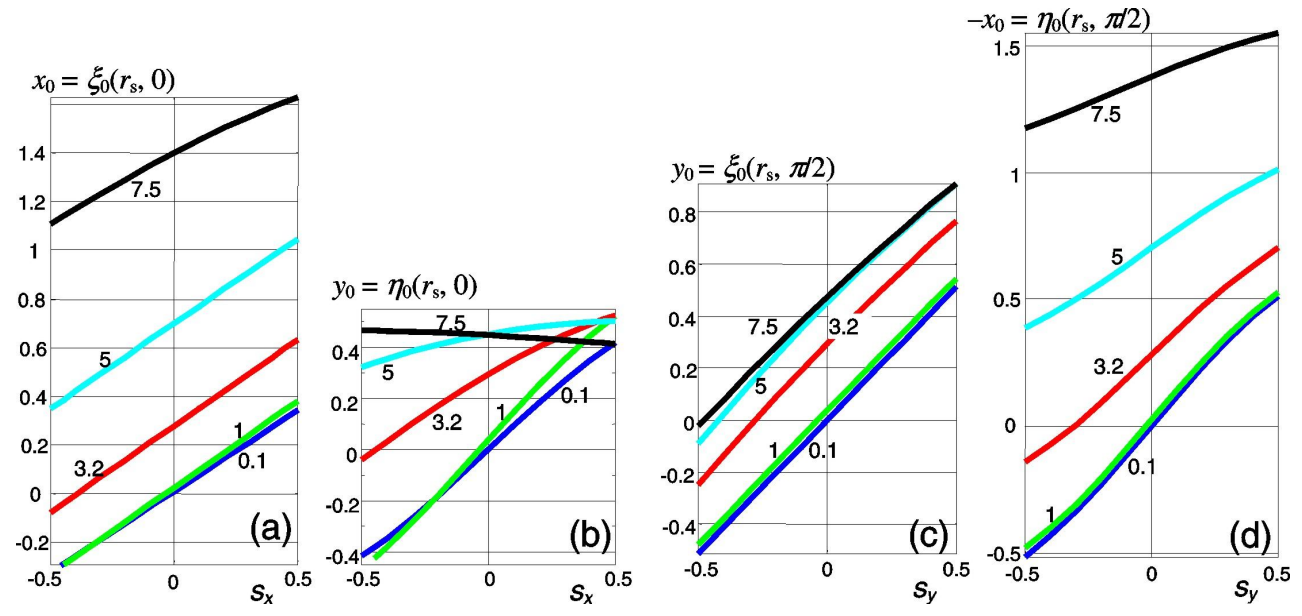

Fig. 6. The same as Fig. 4, but for the extinction coefficient $\kappa=0.4$.

Interesting parallels with the reference case may be revealed if one observes evolution of the diffracted beam under conditions of a fixed $x$-oriented misalignment (see Fig. 8). Just like for thin HEs with the EPSs, the CG component $x_{0}\left(z_{2}\right)$ in the plane of misalignment is constant (see horizontal lines in Fig. 7a-c); small deviations in the initial segments of the horizontal lines 1 and 5 in panel (c) can be attributed to a ripple structure which represents a characteristic feature of the HEgenerated OV-beams at small propagation distances [32, 33, 36, 37]. However, the $x_{0}$ value differs from $s_{x}$ and, generally, it depends on the HE structure. When comparing the panels (a), (b) and (c), one can see that the main factor of this dependence is light extinction in the HE depth. Its growth provokes initially a slight and then a pronounced difference in the $x_{0}\left(z_{2}\right)$ behaviours for different HE thicknesses.

The linear growth of the CG components $y_{0}\left(z_{2}\right)$ orthogonal to the misalignment vector (see oblique lines in Fig. 8) discloses the 'angular' character of transformation of the diffracted beam: 
the CG trajectory behaves as if it experienced 'rotation' around the misalignment axis (cf. with the similar feature for thin HEs with EPS [33]). Note that increase in the extinction is accompanied by increasing steepness of the $y_{0}\left(z_{2}\right)$ dependences: the lines marked as 1 are almost identical in Fig. 8a-c, whereas the lines marked as 3.2 and 5 lie below the line 1 in panels (a) and (b), though they are located above it in panel (c) describing the HEs with high extinction coefficients. This agrees well with behaviour of the curves with the same marks in Fig. 5b, Fig. $5 \mathrm{~d}$ and Fig. 6b, Fig. $6 \mathrm{~d}$ corresponding to the fixed diffracted beam section $z_{2}=1$.
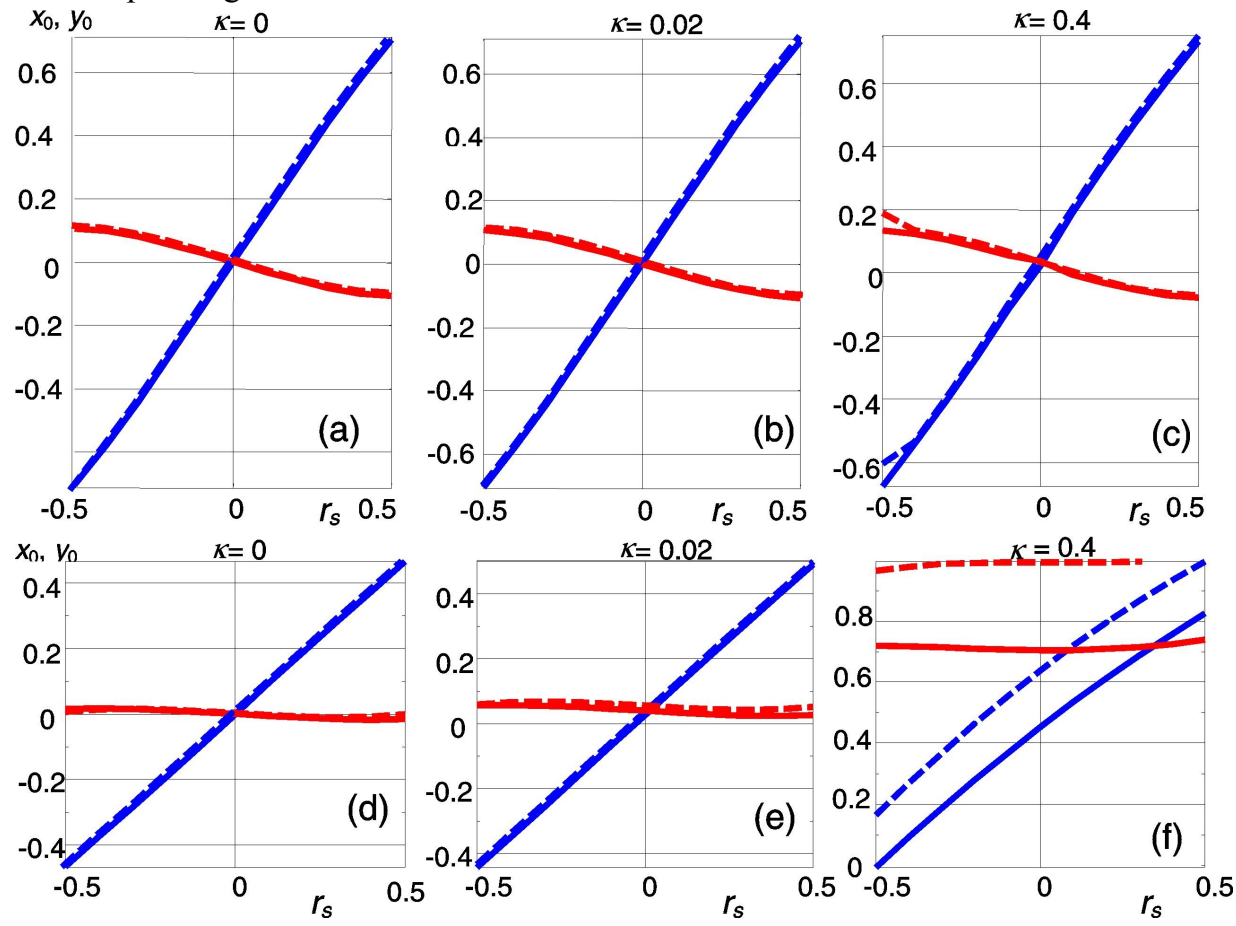

Fig. 7. Coordinates $x_{0}$ (red) and $y_{0}$ (blue) of diffracted-beam CG for the case when the incident beam is shifted along the coordinate bisector $\left(\phi_{s}=\pi / 4\right)$. The HE thicknesses are equal to $2 d=b(\mathrm{a})-(\mathrm{c})$ and $2 d=5 b$ (d)-(f). Solid lines correspond to direct calculations with Eqs. (8) and (14) for $\phi_{s}=\pi / 4$ and dashed lines to the results obtained by composing the CG sensitivities to the orthogonal components of the incident beam shift using the formulae (18). The extinction coefficients in the units of $b^{-1}(6)$ are indicated above each image.
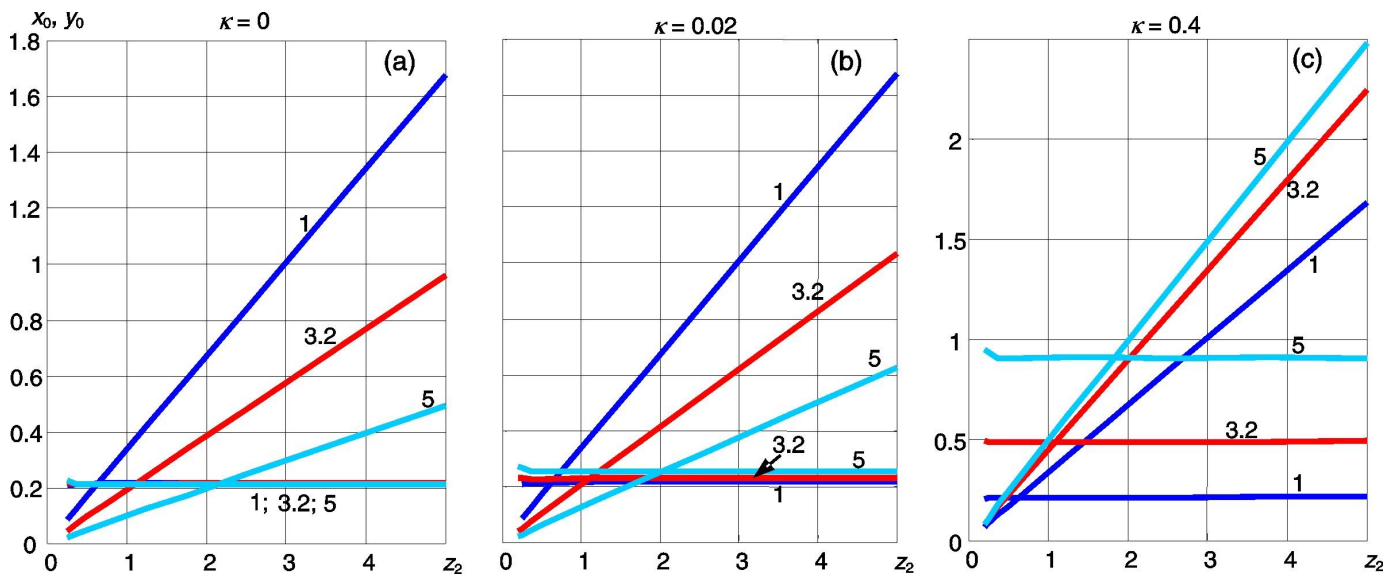

Fig. 8. Evolution of $C G$ coordinates for the case when the diffracted beam propagates behind the HE with the extinction coefficients $\kappa=0$ (a), $\kappa=0.02$ (b) and $\kappa=0.4$ (c). The incident beam misalignment is given by $s_{y}=0$ and $s_{x}=0.3 b$. The functions $x_{0}\left(z_{2}\right)$ are approximately constant (horizontal lines) and the functions $y_{0}\left(z_{2}\right)$ increase linearly (oblique lines). The HE thicknesses are indicated near each line. 


\section{Conclusion}

The results presented above constitute a continuation of the systematic study [37] of volume HEs with the EPS designed for generating the OV-beams. Here we have analyzed theoretically and numerically the consequences of misalignments of the incident beam, especially for the cases when the axis of the incident beam experiences a parallel shift with respect to the nominal HE centre.

Such shifts are inevitable in real transformation schemes and the knowledge concerning their influences is helpful for designing and arranging these schemes. Additionally, like in the case of a thin HE with EPS investigated earlier [32, 33, 36], the misalignments induce controllable transformations of spatial structure of the generated OV-beams, which can be used for deliberately controlling their properties. In particular, we have shown that in the case of a volume HE, purposeful translation of the incident beam axis can compensate distortion of the output beam profile appearing due to light extinction in the HE depth or due to HE thickness and high-angle diffraction [37]. Moreover, it can restore a circularly symmetric OV-beam necessary for different applications.

Along with the overall profile of the diffracted beam, we have considered the behaviour of its $\mathrm{CG}$ as a special and universal characteristic of propagation of the output beam. In agreement with the physics of thin HEs [33], the CG trajectory looks as if it experienced rotation around the vector of misalignment: the $\mathrm{CG}$ component $\xi_{0}$ parallel to the misalignment does not change during the beam propagation, while the orthogonal component $\eta_{0}$ increases linearly. However, in contrast to the case of thin HEs, the exact $\xi_{0}$ and $\eta_{0}$ values depend upon the direction of misalignment $\phi_{s}$ with respect to the horizontal and vertical axes of the volume HE (see Fig. 1). These discrepancies are mainly stipulated by extinction of the incident beam. They become highly articulate if the light extinction in the HE depth is strong enough (see Fig. 6, Fig. 7f and Fig. 8c). For moderate HE thicknesses (up to $2 d \approx b$ ), the OV-beams obtained by the volume HEs with EPS behave in a fair compliance with the theory for the thin CGs developed under high-angle diffraction conditions [36].

The results of this work can be useful for many areas where the OV-beams are applicable. Especially, they would be practical for metrological purposes when the sensitivity of the diffracted OV-beam (in particular, its CG component $\eta_{0}$ ) to the misalignment of the incident beam can be used for detecting and measuring small spatial shifts of the HE with EPS [33, 44] and, intermediately, small shifts and deformations of remote objects $[9,13]$. However, the results obtained by confronting different curves presented in the right columns of Figs. 4-6 imply that the volume character of HEs associated with their thicknesses and the extinction in the HE depth can only decrease misalignment sensitivity of the diffracted-beam CG down to its apparent disappearance (see curves 5 and 7.5 in Fig. 6b). On the other hand, this observation allows one to expect that the HE thickness can be employed for increasing spatial stability of the generated OV-beam characteristics.

\section{References}

1. Soskin M S and Vasnetsov M V, 2001. Singular optics. Progr. Opt. 42: 219-276.

2. Bekshaev A Ya, Soskin M S and Vasnetsov M V. Paraxial light beams with angular momentum. New York: Nova Science Publishers (2008).

3. Nieminen T A, Higuet J, Knoner G, Loke V L Y, Parkin S, Singer W, Heckenberg N R and Rubinsztein-Dunlop H, 2006. Optically driven micromachines: progress and prospects. Proc. 
SPIE. 6038: 237-245.

4. He H, Friese M E J, Heckenberg N R and Rubinsztein-Dunlop H, 1995. Direct observation of transfer of angular momentum to absorptive particles from a laser beam with a phase singularity. Phys. Rev. Lett. 75: 826-829.

5. Simpson N B, Dholakia K, Allen L and Padgett M J, 1997. Mechanical equivalence of spin and orbital angular momentum of light: an optical spanner. Opt. Lett. 22: 52-54.

6. Masajada J, Leniec M, Drobczyński S, Thienpont H and Kress B, 2009. Micro-step localization using double charge optical vortex interferometer. Opt. Express. 17: 16144-16159.

7. Masajada J, Leniec M, Jankowska E, Thienpont H, Ottevaere H and Gomez V, 2008. Deep microstructure topography characterization with optical vortex interferometer. Opt. Express. 16: 19179-19191.

8. Wang W, Yokozeki T, Ishijima R, Takeda M and Hanson S G, 2006. Optical vortex metrology based on the core structures of phase singularities in Laguerre-Gauss transform of a speckle pattern. Opt. Express. 14: 10195-10206.

9. Anzolin G, Tamburini F, Bianchini A and Barbieri C, 2009. Method to measure off-axis displacements based on the analysis of the intensity distribution of a vortex beam. Phys. Rev. A. 79: 033845 .

10. Mawet D, Riaud P, Absil O and Surdej J, 2005. Annular groove phase mask coronagraph. Astrophys. J. 633: 1191-1200.

11. Swartzlander G A, Jr, 2009. The optical vortex coronagraph. J. Opt. A. 11: 094022.

12. Tamburini F, Umbriaco G, Anzolin G, Barbieri C, Bianchini A, 2006. FrogEye, the quantum coronagraphic mask. The photon orbital angular momentum and its applications to astronomy. Mem. Soc. Astron. Ital. Suppl. 9: 484-485.

13. Anzolin G, Tamburini F, Bianchini A, Umbriaco G and Barbieri C, 2008. Optical vortices with starlight. Astron. Astrophys. 488: 1159-1165.

14. Gibson G, Courtial J, Padgett M, Vasnetsov M, Pas'ko V, Barnett S and Franke-Arnold S, 2004. Free-space information transfer using light beams carrying orbital angular momentum. Opt. Express. 12: 5448-5456.

15. Karimi E, Piccirillo B, Nagali E, Marrucci L and Santamato E, 2009. Efficient generation and sorting of orbital angular momentum eigenmodes of light by thermally tuned $q$-plates. Appl. Phys. Lett. 94: 231124.

16. Slussarenko S, Karimi E, Piccirillo B, Marrucci L and Santamato E, 2009. Universal unitary gate for single-photon spin-orbit four-dimensional states. Phys. Rev. A. 80: 022326.

17. Fadeyeva T, Rubass A, Volyar A and Swatzlander G, 2008. Qudrefringence of optical vortices in uniaxial crystal. J. Opt. Soc. Amer. A. 25: 1643-1641.

18. Marrucci L, Karimi E, Slussarenko S, Piccirillo B, Santamato E, Nagali E and Sciarrino F, 2012. Spin-to-orbital optical angular momentum conversion in liquid crystal q-plates: classical and quantum applications. Mol. Cryst. Liq. Cryst. 561: 48-56.

19. Skab I, Vasylkiv Yu, Zapeka B, Savaryn V and Vlokh R, 2011. Appearance of singularities of optical fields under torsion of crystals containing threefold symmetry axes. J. Opt. Soc. Amer. A. 28: 1331-1340.

20. Skab I, Vasylkiv Yu, Smaga I and Vlokh R, 2011. Spin-to-orbital momentum conversion via electrooptic Pockels effect in crystals. Phys. Rev. A. 84: 043815.

21. Soroko L M. Holography and coherent optics. New York: Plenum Press, London (1980).

22. Goodman J W. Introduction to Fourier optics. New York: McGrow-Hill (1996). 
23. Solimar L and Cook D J. Volume holography and volume gratings. New York: Academic Press (1981).

24. Basistiy I V, Soskin M S and Vasnetsov M V, 1995. Optical wavefront dislocations and their properties. Opt. Commun. 119: 604-612.

25. Heckenberg N R, McDuff R, Smith C P, Rubinstein-Dunlop H and Wegener M J, 1992. Laser beams with phase singularities. Opt. Quant. Electron. 24: S951-S962.

26. Bazhenov V Yu, Vasnetsov M V and Soskin M S, 1990. Laser beams with screw dislocations in their wavefronts. JETP Lett. 52: 429-431.

27. Rozas D, Law C T and Swartzlander G A, Jr, 1997. Propagation dynamics of optical vortices. J. Opt. Soc. Amer. B. 14: 3054-3065.

28. Sacks Z S, Rozas D and Swartzlander G A, Jr, 1998. Holographic formation of optical-vortex filaments. J. Opt. Soc. Amer. B. 15: 2226-2234.

29. Swartzlander G A, Jr, Optical vortex filaments. In: Optical vortices. Ed. by Vasnetsov M and Staliunas K. New York: Horizons in World Physics 228, Nova Science Publishers (1999).

30. Pas'ko V A, Basistiy I V, Vasnetsov M V and Soskin M S, 2004. Analysis of optical vortex beams with integer and fractional topological charge. Proc. SPIE. 5477: 83-88.

31. Basistiy I V, Pas'ko V A, Slyusar V V, Soskin M S and Vasnetsov M V, 2004. Synthesis and analysis of optical vortices with fractional topological charges. J. Opt. A: Pure Appl. Opt. 6: S166-S169.

32. Bekshaev A Ya and Karamoch A I, 2008. Spatial characteristics of vortex light beams produced by diffraction gratings with embedded phase singularity. Opt. Commun. 281: 13661374.

33. Bekshaev A Ya and Karamoch A I, 2008. Displacements and deformations of a vortex light beam produced by the diffraction grating with embedded phase singularity. Opt. Commun. 281: 3597-3610.

34. Karimi E, Zito G, Piccirillo B, Marrucci L and Santamato E, 2007. Hypergeometric-Gaussian modes. Opt. Lett. 32: 3053-3055.

35. Liu Yi-Dong, Gao Chun-Qing and Gao Ming-Wei, 2008. Study on holographic grating diffraction for Laguerre-Gaussian beam generation, Chinese Phys. B. 17: 1769-1776.

36. Bekshaev A, Orlinska O and Vasnetsov M, 2010. Optical vortex generation with a "fork" hologram under conditions of high-angle diffraction. Opt. Commun. 283: 2006-2016.

37. Bekshaev A Ya, Sviridova S V, Popov A Yu and Tyurin A V, 2012. Generation of optical vortex light beams by volume holograms with embedded phase singularity. Opt. Commun. 285: 4005-4014.

38. Mair A, Vaziri A, Weihs G and Zeilinger A, 2001. Entanglement of the orbital angular momentum states of photons. Nature (London). 412: 313-316.

39. Leach J, Jack B, Romero J, Ritsch-Marte M, Boyd R W, Jha A K, Barnett S M, FrankeArnold S and Padgett M J, 2009. Violation of a Bell inequality in two-dimensional orbital angular momentum state-spaces. Opt. Express. 17: 8287-8293.

40. Molina-Terriza G, Torres J P and Torner L, 2003. Orbital angular momentum of photons in noncollinear parametric downconversion. Opt. Commun. 228: 155-160.

41. Osorio C I, Molina-Terriza G and Torres J P, 2009. Orbital angular momentum correlations of entangled paired photons. J. Opt. Am. 11: 094013.

42. Abramowitz M and Stegun I, Eds. Handbook of mathematical functions. National Bureau of Standards. Applied Mathematics Series, \#55 (1964).

Ukr. J. Phys. Opt. 2013, Volume 14, Issue 4 
43. Bekshaev A Ya and Karamoch A I, 2008. Astigmatic telescopic transformation of a highorder optical vortex. Opt. Commun. 281: 5687-5696.

44. Bekshaev A Ya and Sviridova S V, 2010. Effects of misalignments in the optical vortex transformation performed by holograms with embedded phase singularity. Opt. Commun. 283: 4866-4876.

Bekshaev A., Sviridova S., Popov A., Rimashevsky A. and Tyurin A. 2013. Optical vortex generation by volume holographic elements with embedded phase singularity: Effects of misalignments. Ukr.J.Phys.Opt. 14: $171-186$.

Анотація. На основі лінійної теорії формування оптичних вихорів (OВ) в об'ємних голографічних елементах (ГЕ) із вбудованою фазовою сингулярністю (A. Bekshaev et al., Opt. Cоттип. 285 (2012) 4005) теоретично аналізуються ОВ-пучки, одержані за умов, коли падаючий гаусівський пучок відхиляється від оптичної осі ГЕ. Засобами чисельного аналізу розглянуто просторові характеристики та еволючію дифрагованих пучків з урахуванням екстинкиії падаючого випромінювання в товщі ГЕ. Особливу увагу надано поведінці центра тяжіння дифрагованого пучка. Показано, щзо завдяки зсувам падаючого пучка відносно осі ГЕ з'являється можливість иілеспрямовано керувати формою одержаного вихрового пучка, зокрема, для компенсації спотворень його профілю, обумовлених поглинанням та екстинкиієєю в ГЕ.

M. Abramowitz and I. A. Stegun (Eds.) (1964) Handbook of Mathematical Functions with Formulas, Graphs, and Mathematical Tables, National Bureau of Standards Applied Mathematics Series,

U.S. Government Printing Office, Washington, D.C.. 